\title{
La construcción de una tipología de segmentación del mercado de trabajo
}

\author{
Pedro López Roldán
}

Universitat Autònoma de Barcelona. Departament de Sociologia.

08193 Bellaterra (Barcelona). Spain

\section{Resumen}

El objetivo de este arrículo consiste en aplicar un diseño de análisis que sea adecuado a la concepción de la denominada tipologia estructural y articulada. Se trata de un diseño basado en la utilización de la encuesta y de técnicas de análisis multivariable, dirigido a contrastar la hipótesis central de la perspectiva teórica de la segmentación del mercado de trabajo por medio de un modelo teórico basado en su conceptualización desde el empleo. El análisis expuesto toma como referente espacial y temporal el del mercado de trabajo delimitado por la Regió Metropolitana de Barcelona para cl año 1990 y en base a la información recogida en la Enquesta de la Regió Metropolitana de Barcelona sobre condicions de vida i bábits de la població.

Palabras clave: metodología, cipología, segmentación del mercado de trabajo, modelos, diseño de análisis, análisis de correspondencias, análisis de clasificación.

Abstract. The Constrution of a labour market segmentation typology

The objective of this article is to apply an analysis design useful to the ustructural and articulated typologiesn, that is, a design based on survey and multivariate statistical methods for collecting and analyzing data which leads to contrast the main hypothesis proposed from the theoretical perspective of the labor market segmentation. The research has a geographic and temporal reference: the labor market delimited by the Metropolitan Region of Barcelona in 1990, and analyze survey data designed and collected in the called Enquesta de la Regió Metropolitana de Barcelona sobre condicions de vida $i$ habits de la població.

Key words: methodology, typology, labour market segmentation, models, research design, correspondence analysis, cluster analysis.

\section{Sumario}

1. Presentación: una problemática teórica y metodológica

2. Restulrados del análisis
3. Comentarios finales

Bibliografía 


\section{Presentación: una problemática teórica y metodológica}

El objetivo de este artículo consiste en presentar el proceso y los resultados de la aplicación de un diseño de análisis destinado a la construcción de una tipología de segmentación del mercado de trabajo. Partiendo de la distinción entre la forma y el contenido del objeteto de investigación, por un lado, este diseño se considera homólogo o adecuado formalmente a lo que hemos denominado modelo metodologico de una tipologia estructural y articulada, esto es, un modelo general que reconoce en el objero de estudio una morfología tipológica de naturaleza estructural y articulada ${ }^{l}$. Por otra parte, y entendiendo que el método no puede disociarse de los objetos que se investigan, una problemática de estudio nos mostrará el contenido sustantivo de la morfologia tipológica. Esta problemática es la que se deriva de conceptualizar el funcionamiento del mercado de trabajo desde la perspectiva teórica de la segmentación.

\section{El contenido sustantivo de la segmentación}

La explicación del funcionamiento del mercado laboral desde la segmentación, en contraposición con la visión neoclásica, se traduce en la estructuración de un mercado de trabajo no homogéneo y dividido en segmentos diferenciados e interrelacionados jexárquicamente según procesos de internalización-externalización y en donde confluyen diversos factores de caracterización. Esta es la hipótesis central y genérica que resume e identifica las distintas aportaciones teóricas que desde los años sesenta han razonado los economistas y los sociólogos segmentaristas ${ }^{2}$.

El tratamiento que aquí realizamos de esta problemática se construye a partir de varios elementos. En primer término, se adopta un enfoque que parte de un supuesto principal: la segmentación es el resultado del proceso social de trabajo en la empresa que estructura los distintos puestos de trabajo. Por tanto, el fenómeno de la segmentación se entiende en el contexto de las relaciones sociales de producción, en la esfera de la producción, y se centra en los aspectos de la demanda del mercado de trabajo. En segundo término, nuestra aproximación al fenómeno de la segmentación se realiza desde una perspectiva del empleo, en la esfera de la circulación, entendiendo que el proceso de segmen-

1. Esta concepción de la tipologia se desarrolla en otro artículo del presente número de la revista.

2. Nuestro modelo tebrico de segmentación se elabora a partir de distintas constribuciones teóricas que han aportado diversos autores y autoras, entre ellos: institucionalistas (M.I. Ptore, P.B. Doeringer), radicales (D.M. Gordon, R.C. Edwards, M. Reich), de la escuela de Cambdridge (F. Wilkinson, J. Rubery, P. Villa) o de la literacura francesa (M. Maruani, F. Michom, M. Maurice, P. Desmarez). No obstante, la perspectiva adoptada parte sobre todo de la tradición de pensamiento marxista que se expresa en los trabajos de la escuela radical americana. El modelo teórico se desarrolla más extensamente en la tesis presentada por el autor del artículo (P. López, 1994) y en una investigación financiada por la DGICYT sobre Transformaciones del Trabajo y Bienestar Social (F. Miguélez, 1989; Grup d'Estudis Sociologics sobre la Vida Quotidiana i el Treball, 1994). 
tación de los puestos de trabajo originado en la empresa tiene su reflejo en formas específicas de empleo de la fuerza de trabajo. Por lo tanto, el mercado de trabajo y las formas y condiciones de contratación de los trabajadores/as asalariados/as que ocupan los distintos puestos de trabajo se convierte en el centro del análisis. En tercer lugar, esta perspectiva nos orienta hacia una visión del fenómeno de la segmentación que podemos calificar como global o macrosociológica que pierde el referente de la empresa como centro del análisis. Por lo tanto, la construcción del modelo de segmentación se destina a mostrar los vínculos existentes entre la esfera de la producción y la esfera de la circulación, a la relación que se establece entre trabajo y empleo. En cuarto lugar, se concibe que el proceso de segmentación responde a explicaciones que van más allá de los factores estrictamente productivos, destacando factores sociales de origen de la fuerza de trabajo potencialmente activa, por tanto, factores ligados a la estructura social y a la reproducción social ${ }^{3}$.

Esta línea de investigación nos permite avanzar en algunos aspectos del tratamiento de la problemárica de la segmentación. Por un lado, y recogiendo la necesidad de ofrecer una visión contextual del proceso de segmentación, se trata de contrastar la hipótesis fundamental de fragmentación del mercado de trabajo para la Regió Metropolitana de Barcelona, una extensa zona geográffca cuya dinámica económica, laboral y social ofrece un observatorio privilegiado para constatar la presencia y la magnitud de este tipo de fenómeno. Por otro, esta visión contextual ofrece una panorámica que es la del conjunto de la economía de esre área y, por tanto, se extiende más allá de los tradicionales estudios del sector industrial, incluyendo la actividad de los servicios así como fenómenos de economía sumergida que los estudios habiruales no recogen. Por último, esta aproximación extensiva se plantea desde una visión distinta a la de la mayor parte de los estudios sobre la segmentación, pues toma como unidad de análisis a la población asalariada que ocupa un puesto de trabajo, lo que nos permite adoptar una visión basada en el empleo donde se conjugan fundamentalmente aspectos determinantes de la demanda de trabajo con otros también de caracterización social de la oferta.

El análisis que presentamos en estas páginas busca validar la hipótesis central que ha puesto de manifiesto la perspectiva de la segmentación: la configuración de una tipología de segmentos o de situaciones de empleo que estructuran un mercado laboral definido por la división del empleo asalariado en dos segmentos, un segmento primario y otro secundario, el primero de los cuales comprende, a su vez, la distinción entre un segmento primario independiente y otro dependiente. Esta hipótesis se deriva del concepto de segmentación donde cabe reconocer la posición de dominación de los empre-

3. Esta apreciación sobre la esfera de la reproducción amplia teóricamente el análisis de la realidad del trabajo productivo, asi como de su ausencia, mostrando la relevancia de los factores ligados a la oferta de trabajo. No obsrance, si bien esta referencia es tenida en cuenta en la invesrigación al consruir el concepto de segmentación desde una perspectiva de! empleo, el planteamiento tiene su foco de atención en los procesos derivados del lado de la demanda. 
sarios, la acción de los trabajadores y el papel del Estado. Las diversas estrategias implicadas y el concepto de mercado interno de trabajo nos conducen a determinar la relevancia de cuatro dimensiones principales con las que caracterizar los distintos segmentos de empleo. Tres de eflas se engloban como dimensiones de caracterización de los puestos de trabajo en términos de empleo: la estabilidad laboral, como dimensión central, la cualificación efectiva ${ }^{4}$ y el salario. Una cuarta se refiere a la caracterización o contextualización de las empresas incluyendo el tamaño de los centros de trabajo, el sector de actividad, su titularidad y la presencia de representación laboral.

La interrelación o combinación de estas dimensiones nos permite mostrar la naturaleza del proceso de segmentación y la configuración de una tipología de segmentos de empleo que estrucrura el mercado de trabajo en empleos diferenciados y jerarquizados. Una primera distinción es la que se establece entre segmento primario y secundario, explicada básicamente por la dimensión de estabilidad-inestabilidad. El segmento primario se caracteriza por recoger las situaciones de empleo más estable característicos de los mercados internos de las grandes empresas y del sector público que se corresponden con puestos de trabajo centrales para la actividad de la empresa. La capacidad de negociación de los trabajadores de este segmento les dota de una garantía de mejores condiciones laborales y la regulación de las mismas, con claros mecanismos de promoción. El segmento secundario se definiria por la inestabilidad del empleo resultado de las estrategias de externalización que configura empleos con bajos niveles de cualificación y que no posibilitan su adquisición, con malas condicones laborales y localizados en pequeñas empresas donde se carece de capacidad de negociación. En este segmento cabría incluir también el fenómeno de la economía sumergida. Una segunda división se establece en el interior del segmento primario resultado de estrategias de flexibilización y control de la fuerza de trabajo. Esta división tiende a diferenciar un segmento primario independiente, de empleo estable con los mayores niveles de cualificación, mejor remunerados y más enriquecedores en correspondencia con la centralidad de los puestos de trabajo que se ocupan. El segmento primario dependiente contempla los puestos estables de menor cualificación que implican tareas más rutinarias y específicas, y el mantenimiento de unas buenas condiciones de trabajo que resulta de una capacidad de negociación de sus trabajadores.

\section{El contenido formal tipologico}

La aplicación de un diseño de análisis pertinente a esta problemática nos conducirá a establecer la hipótesis de segmentación laboral. Este diseño, adecuado para el modelo teórico que se ha construido, lo es también para el modelo formal tipológico inherente al objeto: se trata de obtener una tipología estrtuctural y articulada. Esta correspondencia entre forma y contenido se expresa en diferentes aspectos.

4. Sobre el concepto de cualiftcación efecriva, ver A. Lope (1994). 
En primer lugar, se concibe desde el modelo de la segmentación una ordenación de situaciones de empleo internamente relacionadas para el conjunto del empleo asalariado. Se trata, por tanto, de un modelo de estructuración de situaciones de empleo que da lugar a una tipología de segmentos de empleo.

Un segundo aspecto es que esta estructuración se contempla desde una visión global o contextual, por lo que se añade el carácter de generalizador. Este aspecto es menos relevante desde el punto de vista del carácter de estos modelos, aunque sí lo sea desde el objetivo de validación externa. El modelo de segmentación se ha concebido a través del empleo y en un conjunto de unidades, la población asalariada, a la que sistemáticamente se les atribuye e identifica de antemano con una serie de características que nos dan idea dc ciertos comportamientos y situaciones de hecho, estandarizables, translingüísticos e inicialmente desestructurados. Este es el proceder de una perspectiva metodológica de investigación atributiva o distributiva (J. Ibáñez, 1985), es el proceso que nos lleva a hablar de explicación estructural a través de interrelación del conjunto de variables identificativas del fenómeno de la segmentación.

En tercer lugar, la perspectiva teórica de la segmentación nos ha permitido elaborar un marco conceptual que nos ofrece una aproximación desde las situaciones de empleo. Esta circunstancia nos plantea dos cuestiones de interés: se trata de una nueva elaboración conceptual, que introduce ciertos elementos de incertidumbre en relación a la hipótesis central de la segmentación, pero esta elaboración se traduce en un conjunto explícito y pertinente de dimensiones sobre las que se justifican y se establecen diversas hipótesis de comportamiento que nos aseguran criterios teóricos de combinación de atriburos y de comportamiento previsible. Las preguntas que se formulan en la construcción del modelo de la segmentación entroncan con el carácter del procedimiento de construcción tipológica: ¿con qué criterios operativos podemos establecer la combinación entre los distintos indicadores de las dimensiones de la segmentación?, ¿en qué medida una conceptualización en función del empleo no introduce elementos de distorsión o aporta nuevos elementos en la configuración de los segmentos según la hipótesis general planteada? La resolución de estas cuestiones se orienta en primera instancia hacia la construcción teórica realizada y también mediante una dinámica de articulación: de contraste e interrelación entre el nivel teórico de los conceptos y sus relaciones y el nivel de las variables y sus relaciones.

Estos comentarios ponen de manifiesto, en cuarto lugar, el carácter conceptual de la tipología estructural y articulada. Estructuración y articulación en cuanto que los conceptos originales, individuales, que han sido seleccionados y justificados en el marco de la perspectiva de la segmentación tienen una traducción final en una tipología, pero al mismo tiempo permite o sugiere la reelaboración conceptual original del fenómeno de la segmentación. Esta forma de proceder introduce un grado de flexibilidad fructífero para la investigación que recoge la práctica habitual de cualquier investigación donde se interrelacionan procesos deductivos e inductivos, de análisis y validación. En este sentido, los tipos de la tipología de segmentación darán lugar a una 
definición asociada a ese proceso flexible de articulación, por intensión y extensión.

Por último, esta definición de los tipos que incorpora el carácter conceptual de la tipología permite la comparación entre ellos $\mathrm{y}$, consiguientemente, la diferenciación y ordenación de las distintas situaciones de empleo, porque es el objetivo conceptual y porque técnicamente eso se pretende según un diseño de análisis apropiado. La diferenciación y la ordenación, en una perspectiva atriburiva, conducen por tanto a la medición del fenómeno de la segmentación, en términos de una variable de naturaleza cualitativa, nominal u ordinal, inherente en el propio concepto de segmentación.

En síntesis, el modelo metodológico de la tipología estructural y articulada nos proporciona un esquema abstracto y simplificado que refleja una forma habitual de los objetos de estudio sociológicos y que aquí se muestra como homólogo al modelo de segmentación laboral.

\section{El diseño de análisis}

El diseño de análisis, como dispositivo operativo de métodos y técnicas de análisis de datos, ha de ser homólogo con relación al modelo de la tipología articulada y estructural y con relación al modelo de segmentación del empleo, además de incorporar los elementos de análisis y la validación de la hipótesis de segmentación del empleo. Este diseño de análisis se organiza a partir de la distinción de dos momentos diferentes, sucesivos, complementarios y recurrentes destinados a la estructuración del fenómeno bajo una forma tipológica. En primer lugar, una etapa de dimensionalización destinada a establecer una estructura inicial del espacio de atributos que delimita la tipología en su campo conceptual por medio de la combinación y la reducción de las variables originales. En segundo lugar, una etapa de clasificación destinada a constituir los tipos de la tipología como conjunto reducido de categorías exhaustivas, excluyentes, homogéneas en su interior y heterogéneas entre ellas que agrupan a las distintas unidades, completando así la estructuración del fenómeno estudiado. Vinculadas a estos dos momentos centrales cabe hablar de otras dos etapas: una previa destinada al tratamiento descriptivo más elemental de la información recogida a través de análisis frecuenciales y de relación bivariable, destinada a preparar el análisis multidimensional, y otra vinculada con todas las anteriores de validación de la información y de los análisis realizados.

La elección de este diseño se justifica y es coherente con los objetivos de construcción tipológica de forma articulada y estructural por diversas razones. En primer lugar, la complejidad de los conceptos tipológicos incorpora una multiplicidad de aspectos o dimensiones cuya interacción o combinación explica la globalidad del fenómeno que se estudia. El análisis de dimensionalización permite operar este proceso a partir de la obtención de aquellas dimensiones subyacentes y significativas que explican las relaciones entre las variables originales. Por tanto, permite la estructuración multidimensional del concepto 
tipológico que inicialmente aparece desestructurado al disponer las variables de forma individualizada.

En segundo término, este proceso implica tres operaciones fundamentales: selección, combinación y reducción, todas ellas referidas a las propiedades de las unidades, del espacio de arributos, y entendidas en un proceso analítico de articulación entre teoría y realidad empírica. La operación de selección es propia del nivel teórico y de la construcción del objeto de estudio, mientras que las de reducción y combinación se materializan de forma articulada, tanto en la aplicación de un instrumento estadístico como en la interpretación y validación de los resultados cuya pertinencia, además de técnica, se establece por aquello que conceptual o teóricamente se ha construido.

El análisis de dimensionalización, en tercer lugar, se opera a través de las técnicas de análisis factorial. Entre ellas, elegimos el análisis de correspondencias múltiples por diversas razones, entre ellas: porque constituye una técrica de interrelación entre variables donde no se establecen a priori relaciones de dependencia entre ellas; porque permite el tratamiento de variables cualitativas medidas a nivel nominal; porque ofrece una formalización geométrica que permite el paso de lo cualitarivo a lo cuantitativo pero conservando la naturaleza y las operaciones propias de las mediciones cualitativas, es decir, se pretende básicamente diferenciar o establecer un orden, con posiciones relativas en un espacio geométrico más que distribuciones precisas de frecuencias o magnitudes; porque permite reconocer relaciones de tipo no lineal; porque permite el establecimiento de diversos procedimientos de validación y estabilidad de los resultados; porque puede complementarse con otras técnicas, en particular, con las de clasificación automática.

Como resultado de este análisis obtenemos una estructuración inicial del fenómeno estudiado a partir de su espacio de atributos. Por ello, en cuarto lugar, la estructuración final se obtiene por el procedimiento de clasificación a partir del espacio de atributos reducido. La aplicación de las llamadas técnicas de clasificación automática exige, por la diversidad de decisiones implicadas, una referencia constante a procedimientos de validación de los resultados que se obtienen, tanto técnicos como sobre todo de significación teórica que guíen el análisis. Por ello esta técnica expresa y exige una dinámica de articulación teórica y empírica en la obrención y en la interpretación de los resultados. Entre la diversidad de procedimientos técnicos de clasificación aquí es pertinente la elección de un proceso donde se distinguen tres etapas: la partición inicial a través del mérodo de nubes dinámicas, de grupos estables; la agregación jerárquica ascendente de las clases iniciales por el método de mínima pérdida de inercia (ward), y la reasignación de las unidades a partir del método de clasificación no jerárquica de centros móviles. Aquí también hay que incluir diversos procedimientos posibles de validación.

La aplicación de este proceso, en quinto lugar, conduce a la obtención de los tipos de la tipología. Estos tipos incorporan una doble naturaleza: se obticnen por medio de un procedimiento técnico extensivo y el resultado, por los criterios teóricos que lo guian y validan, expresa la intensión del concepto tipo- 
lógico que se construye y da consistencia, coherencia y sentido a la clasificación finalmente obtenida definiendo un conjunto de tipos interrelacionados, homogéneos y diferenciados entre ellos. En definitiva, estructurando el fenómeno estudiado, dando lugar a una explicación de carácter estructural.

Por último, la tipología finalmente obtenida significa la concreción de un conjunto de categorías reducidas dotadas de un nivel de medición nominal u ordinal, por tanto, el diseño de análisis aboca también a la medición del fenómeno estudiado.

\section{Resultados del análisis}

En este apartado se presentarán los principales resultados a los que nos conduce el proceso de construcción y validación de la tipología de segmentación del empleo, de acuerdo con la perspectiva teórica y el diseño de análisis que se han comentado. Esta problemática condujo a la recogida de datos que se incluye en la Enquesta de la Regió Metropolitana 1990 sobre condiciones de vida y hábitos de la población.

La información que se maneja parte de una matriz de datos con 2.051 individuos, la submuestra que corresponde a la población asalariada y un conjunto de 14 variables que definen el espacio de atributos de estructuración del mercado de trabajo. Estas variables surgen de la distinción de cuatro dimensiones principales con las que se caracteriza el empleo desde la perspectiva de la segmentación. En el cuadro adjunto se relacionan las variables incluidas en cada dimensión inicial, con sus respectivas categorías.

Junto a estas variables se consideraron en el análisis, con una finalidad descriptiva, parcialmente explicativa y validativa de las características de los segmentos de empleo, una serie de variables de identificación social: la edad, el sexo, el lugar de residencia, el origen geográfico, la lengua y el nivel educativo.

Tras el análisis descriptivo inicial de estas variables mediante tablas de frecuencia y relaciones bivariables que nos facilita unas primeras conclusiones preparatorias del análisis multivariable, el proceso de construcción de la tipología de segmentación del empleo se realiza medianre un análisis de estructuración inicial del espacio de atributos seleccionado de las catorce variables originales. Es una primera etapa que nos soluciona el problema de cómo establecer, combinar y reducir las relaciones significativas entre las variables originales. Mediante el análisis de correspondencias múltiples obtenemos aquellas nuevas variables, combinación lineal de las primeras, que expresan el orden jerárquico de variablidad de la nube de puntos, lo que nos permitirá identificar y elegir aquellos pocos factores o dimensiones que explican y estructuran las situaciones del empleo asalariado. Los resultados nos permitirán pasar del espacio original de catorce variables - y cincuenta y dos categorías o modalidades asociadas - a otro espacio de dimensión reducida con cinco variables factoriajes que caracterizan a la población asalariada.

La primera dimensión obtenida corresponde a la oposición estabilidadinestabilidad laboral, introduciendo la distinción entre los rasgos caracterís- 
ticos del empleo primario y secundario. Se trata de un primer y principal factor que expresa la dualización del empleo asalariado. La segunda dimensión que se obtiene se identifica fundamentalmente en términos de cualificación efectiva, y diferencia un perfil más cualificado propio del segmento primarto inde-

Tabla I. Dimensiones iniciales, variables y caregorias consideradas en el análisis de la segmentación

\section{Estabilidad}

1.1. Relación laboral-Tipo de contratación: Indefinida / Eventual / Sin contrato.

1.2. Duración del contrato: 1 día- 6 meses / 6 meses-1 año / Más de 1 año / Indefinido.

1.3. Tiempo en la empresa actual: Menos de 2 años / 2-5 años / 5-15 años / Más de 15 años.

1.4. Tiempo en paro en los últimos 5 años: No ha estado / Hasta I año / Más de I año.

2. Cualificación efectiva

2.1. Categoría profesional: Trabajador servicios / Técnico medio / Técnico alto / Obrero no cualificado / Obrero cuatificado.

2.2. Principal exigencia en el trabajo: Iniciativa-conocimientos/Precisión-obediencia / Habilidad manual-resistencia física / Capacidad organización / No determinada.

2.3. Variación de conocimientos técnicos en los últimos 5 años: Más / Igual o menos.

2.4. Variación posibilidades de intervención en el trabajo en los últimos 5 años: Más / Igual o menos.

2.5. Ascenso de categoría en los últimos 3 años: Sí / No.

\section{Ingresos}

Ingresos mensuales netos:

Hasra $60.000 / 60.001-90.000 / 90.001-140.000 /$ Más de 140.000/ NS-NC.

\section{Características de las empresas}

4.1. Tamaño del centro de trabajo:

Hasta 25 rabajadores / 26-100/Más de 100.

4.2. Titularidad de la empresa:

Pública / Privada.

4.3. Representación laboral:

Con representación / Sin representación.

4.4. Subsector de accividad:

Metal / Químicas / Textil-calzado-piel / Electrónica / Otras industrias / Construcción / Comercio-hostelería / Transportes-comunicaciones / Financieras / Administración / Sanidad / Enseñanza / Otros servicios. 
pendiente y otro de menor cualificación propio de un segmento primario dependiente. Consiste, pues, en un factor de diferenciación y jerarquización del empleo estable que surgía en la primera dimensión. Junto a esta distinción se establece otra respecto al empleo más inestable: entre los asalariados que trabajan de forma sumergida y los eventuales de reciente incorporación al mercado laboral.

Junto a estos dos factores centrales que estructuran las diferencias entre la población asalariada, los análisis efectuados nos conducen a considerar tres más, de menor importancia, pero que ayudan a matizar las principales relaciones que dibujan la estabilidad y la cualificación. La tercera dimensión se identifica con relación a la titularidad de la empresa, pero oponiendo el empleo de menor cualificación efectiva de la gran empresa pública frente al empleo más enriquecedor de categorías profesionales intermedias de la pequeña empresa privada. La cuarta dimensión se puede calificar de continuidad-discontinuidad laboral, pues nos segrega trayectorias marcadas por la eventualidad, con la entrada y la salida del mercado de trabajo, de la permanencia en la ocupación y en la misma empresa. Por último, en la quinta dimensión toma peso la categoría profesional para distinguir fundamentalmente el empleo público entre técnicos altos y trabajadores de servicios.

Una vez establecidos estos cincos factores de heterogeneidad de las situaciones de empleo, ahora se trata de conseguir aquellos grupos o clases que a efectos de estructuración del fenómeno de la segmentación den lugar a un conjunto de tipos o segmentos de empleo lo más homogéneos posible internamente, por tanto, lo más heterogéneos entre ellos, según las variables criterio o dimensiones de la segmentación. Partimos de una matriz de datos con 2.051 asalariados y 5 variables factoriales sobre la que procedemos a aplicar un proceso de clasificación dividido en tres etapas según señalamos más arriba.

Tras el proceso de validación técnica y teórica de los resultados obtenidos, el contenido final de las clases o grupos resultantes se expresa en términos de cuatro tipos de la tipología que permiten estructurar la realidad del empleo en la Regió Metropolitana de Barcelona desde la perspectiva de la segmentación. Tres de estos tipos se corresponden con la caracterización del segmento primario, mientras que el cuarto responde a la identificación del segmento secundario. En la figura I se reflejan estos cuatro tipos por su ubicación en los dos principales ejes factoriales o dimensiones de la segmentación. A continuación describiremos el contenido de los segmentos de empleo resultantes.

El primer tipo (31\%) es el que denominamos segmento primario dependiente. Contiene las situaciones de empleo estable, correspondiendo a personas que en su mayoría llevan más de quince años en la empresa y casi todos más de cinco. Aquí confluyen otras dos características definitorias. Por un lado, se trata del tipo de empleo estable con una menor cualificación efectiva. Está constituido por asalariados de categorias profesionales medias y bajas, fundamentalmente de obreros cualificados y no cualificados y trabajadores de servicios, que manifiestan un estancamiento profesional derivado de no haber ascendido de categoría en los últimos años, con una mínima capacidad de con- 
trol del propio trabajo. Mayoritariamente ocupan puestos de trabajo a los que se asocian exigencias poco autónomas, como son la precisión en el cumplimiento de las normas y la obediencia, desarrollando capacidades de habilidad manual y resistencia física. Con todo, la variación de los conocimientos técnicos no adquiere una valoración tan negativa, si bien persiste una mayoría que considera que estos conocimientos no han aumentado en los útimos cinco ańos. Esta moderación del aspecto de los conocimientos en la cualificación efectiva confirma la dinámica propia de un perfil general que se identifica plenamente con la caracterización del segmento primario dependiente, donde en particular se ha destacado el proceso de adquisición de cualificaciones más o menos específicas como resultado de la formación y el aprendizaje en el puesto de trabajo.

Por otro lado, se trata del tipo de empleo que se da en mayor proporción en la empresa mediana y, sobre todo, en la gran empresa. Paralelamente, sc registran los mayores niveles de representación laboral de los trabajadores, lo que permite reforzar las garantías de estabilidad laboral de un empleo de más baja cualificación. Siendo un tipo de empleo coincidente con el que se ha tipificado habitualmente un segmento del sector industrial, aquí podemos constatar cómo se localiza tanto en subsectores de la industria como de los servicios. En particular cabe destacar la presencia diversificada de distintos

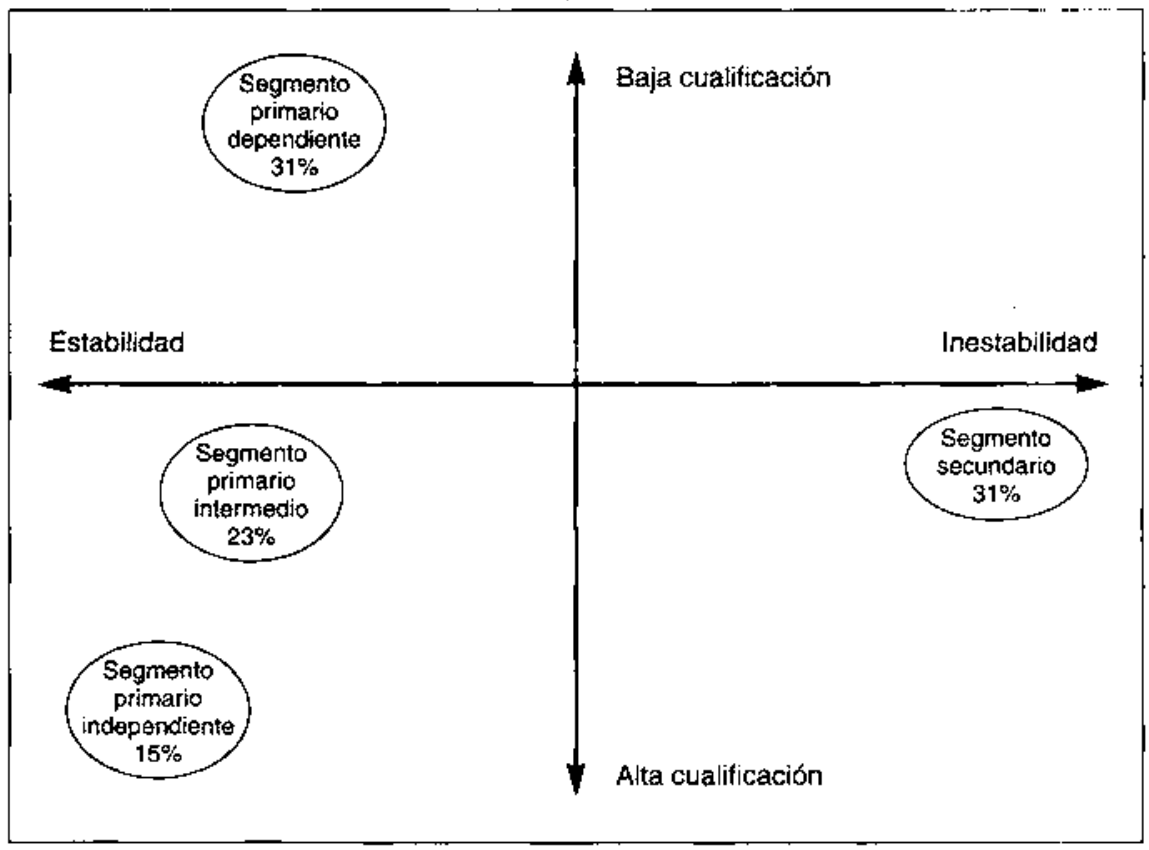

Figura 1 
subsectores, pero sobresalen en mayor medida el metal, químicas, textil/calzado/piel, otras industrias, transportes/comunicaciones y administración, lo cual permite mantener la distribución global de los asalariados entre empresas privadas y públicas.

La caracterización de este tipo o segmento de empleo se completa con una distribución de los ingresos mensuales netos concentrados en los niveles incermedios, entre las 60.000 y las 140.000 ptas. Por otra parte, si atendemos al perfil social de este colectivo, se puede comprobar el predominio de las edades más adultas, por encima de los 40 años de edad, con una mayoría de varones que alcanza el $73 \%$ del total de este primer tipo. El nivel de estudios acabados es el más bajo de todos los asalariados, el $68 \%$ no supera los estudios primarios. El resto de las variables ilustrativas que hemos considerado nos indican la presencia mayoritaria de personas nacidas fuera de Cataluña, que no emplean la lengua catalana, coincidiendo con los procesos inmigratorios que tuvieron lugar en los años sesenta.

Frente a esta definición descriptiva del primer tipo, el segundo (23\%), identificado como segmento primario intermedio, representa una situación de empleo estable e intermedia entre el tipo anterior y el que describiremos a continuación. Intermedio también por su comportamiento en las distintas dimensiones con las que hemos estructurado el espacio de atributos del fenómeno de la segmentación. Desde el punto de vista de la estabilidad, además de recoger el empleo basado en relaciones conrractuales de carácter indefinido y de personas que no han estado desempleadas en los últimos años, este tipo se caracteriza por una vinculación a la empresa menos prolongada en el tiempo de la que aparecía en el primero, concentrándose sobre todo en el período que va de los cinco a los quince años, al mismo tiempo que inclitye una pequeña parte del total de empleo eventual, un 10\%, que lleva más de dos años en la empresa y que guarda las mismas características que describimos seguidamente.

Se trata, en primer lugar, de un tipo que coincide con el primero en la presencia de categorías profesionales intermedias y bajas, pero sobre todo de obreros cualificados, contramaestres y resto de personal administrativo, comercial o técnico, con una proporción también, aunque reducida, de técnicos medios y altos. Esta superior cualificación profesional se ve acompañada por la realización de trabajos que exigen primordialmente iniciativa y conocimientos, así como capacidad de organización. Además, para los empleados que ocupan esos puestos de trabajo ha supuesto, con relación a años anteriores, un proceso de promoción profesional (el $67 \%$ ha ascendido de categoría); y para prácticamente la totalidad de ellos, más posibilidades de intervención y de aplicación de conocimientos técnicos. En segundo lugar, el rasgo que particulariza la naturaleza de este empleo más cualificado es la presencia mayoritaria de la pequeña empresa, hasta veinticinco trabajadores, y en segundo término la de tamaño medio, hasta cien, todas eilas de titularidad privada. Este predominio de pequeña empresa se acompaña también de una ausencia de instancias de representación de los trabajadores que alcanza el $64 \%$ de los empleos de este tipo. Esta realidad laboral de la naturaleza de las empresas se completa con una distri- 
bución de subsectores de actividad que equilibra la distinción entre industria y servicios, pero donde destacan especialmente los del metal, otras industrias, comercio/hostelería y financieras/servicios a las empresas.

Por último, cabe señalar que la distribución de los ingresos de este tipo supera en promedio a los del primero por la mayor proporción de ingresos altos, por encima de las 140.000 ptas. mensuales, aunque mayoritariamente también se concentra en el intervalo de las 60.000 a 140.000 . Comparando este segundo tipo con el primero respecto a las variables de identificación social, se aprecia la coincidencia en el predominio de los varones, pero no así en la distribución de edades, donde, si bien aparecen efectivos de todas ellas, las mayores proporciones se encuentran entre los 25 y los 39 años. Diferente también es el nivel educativo de este colectivo, con un comportamiento similar al del conjunto de los asalariados y que tiende a acentuar los estudios intermedios (formación profesional y bachillerato). La mayor juventud determina a su vez una mayor presencia de nacidos en Cataluña que el primer tipo, y similar a la de los otros dos quse comentaremos. Sin embargo, tanto este segundo tipo como sobre todo el tercero, son los que muestran una mayor familiaridad con la lengua catalana que contrasta con el comportamiento los tipos primero y cuarto.

La consideración del tercer tipo (15\%), el segmento primario independien$t e$, completa la segmentación del empleo más estable y de perfil identificativo del segmento primario que permite establecer una ordenación de las situaciones de empleo que culmina en su nivel superior este tercero. En efecto, se trata del empleo que reúne las mejores condiciones según la evaluación derivada de las distintas dimensiones factoriales.

Coincidiendo con los dos anteriores en la estabilidad del empleo que significa la contratación indefinida, destaca sobre todo la continuidad laboral asociada a llevar de cinco a quince años en la empresa y no haber estado desempleado en los últimos años. También aquí, como sucedía con el segundo tipo, se incluye una pequeña parte del empleo eventual, el $9 \%$ del mismo, que verifica de forma acentuada el perfil de este sercer tipo.

El aspecto más característico que lo identifica es el de la cualificación efectiva, $y$ fundamentalmente por el hecho de que el $84 \%$ se corresponde con la categoría profesional de técnico alto. Consecuentemente, las exigencias relacionadas con este tipo de empleo son de forma predominante las más autónomas, aquellas que requieren iniciativa propia, creatividad o conocimientos. Con semejante intensidad, la experiencia laboral reciente de estos asalariados implica más posibilidades de intervención como de utilización de conocimientos técnicos. Sin embargo, respecto a la promoción laboral, por corresponderse con profesionales que llevan tiempo en la empresa ocupando los niveles de cualificación más altos de la escala laboral, presentan una baja proporción de personas que han ascendido de categoría, sobre todo si se compara con el comportamiento del segundo tipo.

Otro de los aspectos definitorios de este grupo está dado por la combinación de características de las empresas donde trabajan. Se trata sobre todo de asa- 
lariados del sector público, el $67 \%$ está empleado en este tipo de empresa, y conjuntamente con los asalariados de las empresas de titularidad privada concentran su presencia en tres subsectores: enseñanza, administración y sanidad. Esta circunstancia determina la mayor proporción de centros de trabajo de gran tamaño, pero donde también aparecen pequeñas y medianas empresas, siendo globalmente un rasgo común la existencia de representación laboral bajo la forma de comirés o delegados de personal.

Las mayores garantías y mejores condiciones laborales que se dibujan en este tipo se traducen también en la más alta remuneración, en niveles que sobrepasan las 90.000 pras. mensuales a precios del año 1989 . Su caracterización social da lugar a una distribución de edades similar a la del segundo tipo, es decir, con edades situadas entre los 25 y los 44 años. Pero, a diferencia de él, aquí son mayoritarias las mujeres, en una relación con los varones que se reparte entre el 54 y el 46 por ciento, y que se explica por la doble confluencia de empleo público y de subsectores con una importante presencia femenina. Como indicamos anteriormente, se trata también de un tipo con personas nacidas sobre todo en Cataluña, siendo el que en mayor medida utiliza el catalán como lengua habitual y donde se alcanzan los mayores niveles educarivos (el $70 \%$ tiene estudios superiores).

Finalmente, la tipología obtenida de segmentos de empleo se completa con el cuarto tipo (31\%), el segmento secundario, que se opone a todos los anteriores por la dualidad que introduce la primera dimensión de segmentación. En este tipo nos encontramos dos realidades del empleo que se aproximan por los acentuados rasgos de precariedad laboral: el empleo estrictamente eventual y el empleo sumergido. Si bien esta distinción en el interior de este tipo se asocia con ciertos rasgos de diferenciación, constiruyen características menos relevantes en una partición en cuatro tipos que las que marca la separación de un segmento primario o central, con sus diferencias internas, y un segmento secundario de empleo inestable.

De esta forma la definición y especificidad de este tipo viene enfatizada por la inestabilidad que se refleja en las distintas modalidades de situaciones y trayectorias de eventualidad laboral, con excepción de una pequeña proporción de este empleo que se incluye en tipos anteriores. A la eventualidad se vinculan otros rasgos como la baja cualificación efectiva, los bajos ingresos y el predominio de la pequeña empresa privada.

Se trata de un colectivo de asalariados con categorías profesionales medias $y$, sobre todo, bajas, donde destacan los trabajadores de servicios. Los distintos componentes que completan el concepto de cualificación efectiva muestran aquí, en correspondencia con lo que sucede con el primer tipo menos cualificado y de forma algo más acentuada, una experiencia laboral y la realización de tareas marcadas por la ausencia de procesos de recualificación y control del propio trabajo. Asi se pone de manifiesto al observar las principales exigencias asociadas a estos puestos de trabajo, la promoción de categoría, las posibilidades de intervención o de aplicación de nuevos conocimientos. 
La identificación de este último tipo se concreta también por alcanzar los más bajos niveles de remuneración, el $51 \%$ ingresa menos de 60.000 ptas. al mes. Los empleadores de estos asalariados se localizan preferentemente en las empresas de más reducido tamaño, el $62 \%$ corresponde a centros de trabajo de menos de veinticinco trabajadores, donde se alcanzan los más bajos niveles de representación laboral. La titularidad de estos centros de trabajo es privada de forma casi absoluta y destacan principalmente los subsectores de orros servicios (donde se incluye el servicio doméstico), comercio/hostelería, textil/calzado/piel, construcción y otras industrias.

Para acabar la identificación de este tipo señalaremos los rasgos característicos de las variables sociales, algunas ya avanzadas. Presenta una alta proporción de jóvenes, el $57 \%$ comprende el intervalo de dieciocho a veintinueve años y son mayoritariamente -el $54 \%$ - mujeres. Siendo sobre todo de origen caralán, es el grupo que manifiesta una menor urilización del catalán como lengua familiar, correspondiendo por tanto en una buena proporción a la segunda generación de inmigrantes.

\section{Comentarios finales}

La configuración de la estructura del mercado de trabajo de la Regió Metropolitana de Barcelona que se ha presentado permite reafirmar la hipótesis que planteábamos al inicio, pero al mismo tiempo también nos ha aportado resultados de interés como consecuencia del análisis efectuado bajo una conceptualización de la segmentación laboral basada en el empleo. La corroboración de la hipótesis de segmentación ha supuesto identificar una división del empleo asalariado que se estructura en torno a la estabilidad laboral (compaginada con el tamaño de los centros de trabajo, la representación laboral y los ingresos) y la cualificación efectiva. La definición de los tipos o segmentos de empleo ha permitido reconocer la existencia de una variedad ordenada de situaciones de empleo que se corresponden con la caracterización que se afirma desde la perspectiva teórica de la segmentación. Sin embargo, junto al empleo primario (independiente y dependiente) y al empleo secundario, hemos podido constatar la relevancia de un cuarto segmento, intermedio en la división del primario, que muestra la particularidad de la pequeña y mediana empresa para la Regió Metropolitana de Barcelona. Por otra parte, fenómenos específicos como el empleo público y en general de servicios, tan poco tratados en la literatura, o el empleo sumergido, nos han permitido enriquecer los contenidos de un análisis que ha corroborado también, aunque de forma parcial, la existencia de importantes desigualdades sociales.

El análisis del fenómeno de la segmentación pone de maniftesto también la implicación de los distintos elementos del proceso de investigación: el modelo teórico construido, el modelo formal de análisis - la tipología estructural y articulada- y el diseño de análisis, vinculados todos ellos por una hipótesis central que afirma la división y la jerarquización del empleo asałariado en una tipología de segmentos. La interrelación entre todos estos clementos nos per- 
mite hablar de una homología o adecuación entre el contenido de la segmentación laboral y el modelo formal de análisis, y entre éste y el diseño de métodos y técnicas, proporcionando los instrumentos de análisis y validación necesarios de la hipótesis planteada. En definitiva, pues, se concluye la homología o adecuación entre la tipología estructural y articulada como morfología del objeto de estudio para el análisis de la realidad social del empleo.

Semejantes planteamientos pueden ser aplicados en el tratamiento de otras problemáricas teóricas de investigación donde se busque la construcción de una tipología desde una perspectiva metodológica como la aquí presentada. Nada impide tampoco concebir la complementariedad de esta perspectiva con otras más dinámicas, de tipo relacional (análisis de redes), o de estructuras de sentido, que permitan plantear la investigación social desde una aproximación multiestratégica.

\section{Bibliografía}

AL.DENDERFFR, M.S.; BLASHFIELD, R.K. (1987). Cluster Analysis. Beverly Hills: Sage Publications.

AIVIRA MARTfN, Francisco (1989). "Diseños de investigación social: criterios operativos". En: El análisis de la realidad social editado por M. García Ferrando, J. lbáñez y F. Alvira. Madrid: Alianza, p. 85-107.

BAGNASCO, Arnaldo (1989). "Mercado y mercados de trabajo", Sociologia del Trabajo 6, primavera, p. $21-31$.

BENTTEZ, J.; Ruiz, G. (1987). "Segmentación del mercado de trabajo y análisis regional». Información Comercial Española 651, noviembre, p. 56-68.

BenzÉcru, J.P. (1973). L'Analyse des données. Paris: Dunod.

BulbaO, A.; CACHON, L.; PRIETO, C. (1990). Politicas empresariales de mano de obra (Un estudio realizado en la cuenca industrializada del sur madrileño). Madrid: Comunidad de Madrid.

BUTTLER, F.; KEIL, H.J. (1977). «Segmentaciones del mercado de trabajo, polírica del mercado de trabajo y desatrollo económico regional». Revista Española de Economian vol. 1, abrit, p. 43-108.

BISQUERRA AIZINA, Rafael (1989). Introducción conceptual al análisis multivariable. Un enfoque informático con los paquetes SPSS-X, BMDP, LISREL y SPAD. Barcelona: Promociones y Publicaciones Universitarias.

CASSASUS, Cecilia (1980). "Del modelo neoclásico a las teorías de la segmentación del mercado de trabajo". Sociologia del Trabajo 3-4, primera época, p. 9-22.

CORNEJo ÁLVAREZ, Jose Manuel (1988). Técnicas de investigación social: el análists de correspondencias (Teoria y Práctica). Barcelona: Promociones y Publicaciones Universitarias.

Chandon, J.L.; Pinson, S. (1981). Analyse gypologique. Théories et applications. París: Masson.

DOERINGER, P.B.; PIORE, M.J. (1985). Mercados internos de trabajo y análisis laboral Madrid: Ministerio de Trabajo y Seguridad Social.

Edwaros, R.C.; GoRdon, D.M.; REICH, M. (eds.) (1975). Labor Market Segmentation. Lexington (Massachusets): Lexington Books. 
ESCOFIER, B.; PAGES, J. (1988). Analyses factorielles simples et multiples. París: Dunod. FERNÁNDEZ SANTANA, O. (1991). "El análisis de cluster: aplicación, interpretación y validación". Papers. Revista de Sociologia 37, p. 65-76.

GORDON, D.M.; EDWARDS, R.C.; REICH, M. (1986). Trabajo segmentado, trabajadores divididos. La transformación histórica del trabajo en los Estados Unidos. Madrid: Ministerio de Trabajo y Seguridad Social.

GRUP D'ESTUdIS SOCIOLOGICS SOBRE LA VIDA QUOTIDIANA I EL IREBALL (1994). Transformaciones del trabajo y bienestar social. Informe del proyecto de investigación financiado por la DGICYT.

LEBART, Ludovic (1977). "La validité des résultats en analyse des données". Consommation 1, enero-marzo, p. 41-69.

LFBART, L.; MORINEAU, A.; FENELON, J.P. (1985). Tratamiento estadístico de datos Métodos y programas. Barcelona: Marcombo.

LEFEBVRE, Jacques (1983). Introduction aux analyses statistiques multidimensionnelles. Paris: Masson.

LeBART, L.; MorineaU, A.; LAMBert, T. (1987). SPAD. N: Système Portable pour l'Analyse des Données. Version 1.O. Manuel de Référence. Sèvres: Cisia.

LOPE PEÑA, Andreu (1994). Innovación tecnologica y cualificación: la tendencia a la polarización de las cualificaciones en la empresa. Tesis doctoral. Bellaterra: Universizar Aurònoma de Barcelona.

Lorez ROLDÁN, Pedro (1994). La construcción de tipologias en Sociologia: propuesta metodológica de construcción, análisis y validacion. Aplicación al estudio de la segmentación del mercado de trabajo en la Regió Metropolitana de Barcelona. Tcsis doctoral. Bellaterra: Universitat Autònoma de Barcelona.

LOzARES COLINA, C.; LOPEZ ROLDÁN, P. (1991a). "El análisis multivariado: definición, criterios y clasiftcación". Papers. Revista de Sociologia 37, p. 9-29.

- (199 Ib). «El análisis de componentes principales: aplicación al análisis de datos secundarios". Papers. Revista de Sociologia 37, p. 31-63.

- (199Ic). "El muestreo estratificado por análisis multivariado". En: El pluralismo metodológico en la investigación social: ensayos típicos, editado por $\mathrm{M}$. Latiesa. Granada: Universidad de Granada, p. 107-160.

MarTín ARTII.es, Antonio (1992). "Proceso de flexibilización y modelo de relaciones laborales postfordista. Estudio comparado de casos". Sociologia del Trabajo 15, primavera, p. 63-90.

Maruani, M.; ReYNaUd, E. (comp.) (1991). Debates sobre el empleo. Francia y Alemania. Madrid: Ministerio de Trabajo y Seguridad Social.

Migutiez LoBo, Faustino (1989). La segmentación del mercado de trabajo. Mimeo.

Miguélez Lobo, F; Lopez Rolóín, P. (1988). Enquesta Metropolitana 1986. Condicions de vida i hábits de la població de làrea metropolitana de Barcelona. La pobresa a l'area metropolitana de Barcelona. Barcelona: Institut d'Estudis Metropolitans de Barcelona.

- (1989). "El trabajo en la pequeña empresa española. ¿Una perspectiva alternativa?». Papers. Revista de Sociologia 32, p. 63-87.

Micuélez Lobo, F; PRieto Rodriguez, C. (1991). Las relaciones laborales en España. Madrid: Siglo XXI.

Miguélez Lobo, F; Torns Martín, T. (1992). Enquesta de la Regió Metropolitana de Barcelona 1990. Treball, condicions econòmiques i formes de consum. Barcelona: 
Mancomunitat de Municipis de l'Area Metropolitana de Batcelona y Diputació de Barcelona.

Osterman, Paul (comp.) (1988). Los mercados internos de trabajo. Madrid: Ministerio de Trabajo y Seguridad Social.

PIORE, M.J.; SABEL, Ch. (1990). La segunda ruptura industrial. Madrid: Alianza.

PrIETo RodRiguez, Carlos (1989). "¿Mercado de trabajo?», Revista Española de Investigaciones Sociologicas 47, p. 177-192.

ReCro, Albert (1988). Capitalismo y formas de contratacion laboral Madrid: Ministerio de Trabajo y Seguridad Social.

SÁNCHEZ CARRIÓN, Juan Javier (1984). Introducción a las técnicas de análisis multivariable aplicadas a las ciencias sociales. Madrid: Centro de Investigaciones Sociológicas.

Santillana del Barrio, I.; Maycas Tarascon, J. (1979). «La división del metcado de trabajo: el problema de los desaventajadosm. Cuadernos de Ciencias Económicas y Empresariales 5, p. $17-43$.

SENGENBERGER, Werner (comp.) (1988). Lecturas sobre el mercado de trabajo en la República Federal de Alemania. Madrid: Ministerio de Trabajo y Seguridad Social.

TOHARIA, Luis (comp.) (1983). El mercado de trabajo: Teorias y aplicaciones, Madrid: Alianza.

TORNS MARTIN, Teresa (1991). "Trabajo, mujer y clase obrera". En: Trabajo y clase obrera hoy. Fundación de Investigaciones Marxistas.

Torns Martin, T.; Carrasquer Oto, P. (1987). «Entorn dels conceptes de dona i trebail a Catalunya». En: Visió de Catalunya. Barcelona: Diputació de Barcelona, p. 235-247.

VILLA, Paola (1990). La estructuración de los mercados de trabajo. La siderurgia y la construcción en Italia. Madrid: Ministerio de Trabajo y Seguridad Social.

VOLLE, Michel (1985). Analyse des données. París: Economica.

WILKINsON, Frank (ed.) (1981). The Dynamics of Labour Market Segmentation. Londres: Academic Press. 